\title{
Corneoconjunctival manifestations of lymphoma in three dogs
}

\author{
Seonmi Kang ${ }^{1}$, Manbok Jeong ${ }^{2}$, Kangmoon Seo ${ }^{1, *}$ \\ ${ }^{I}$ Department of Veterinary Clinical Sciences, College of Veterinary Medicine and Research Institute for Veterinary Science, Seoul National University, Seoul \\ 08826, Korea \\ ${ }^{2}$ I Animal Medical Center, Bucheon 14427, Korea
}

\begin{abstract}
An 8-year-old Shih Tzu, a 5-year-old Maltese, and a 10-year-old Maltese presented with conjunctival hyperemia and peripheral corneal edema. Severe conjunctival thickening with varying degrees of corneal extension was observed. Cytological examination showed many large lymphocytes with malignant changes in the conjunctiva which was consistent with findings in fine-needle aspiration samples taken from regional lymph nodes. They were diagnosed as having Stage V multicentric lymphoma. When conjunctival thickening is observed in canine patients with multicentric lymphoma, conjunctival metastasis with infiltration of neoplastic lymphoid cells should be included in the differential diagnosis.
\end{abstract}

Keywords: Canine; Conjunctiva; Cornea; Multicentric lymphoma; Uvea

Lymphoma is a common neoplasm in dogs, with an estimated incidence in the range of 13 to 24 per 100,000 dogs at risk and accounts for approximately $7 \%$ to $24 \%$ of all neoplasia and $83 \%$ of all hematopoietic tumors in dogs $[9,13]$. Multicentric lymphoma is the most common type of lymphoma in dogs and accounts for approximately $80 \%$ of cases.

The eye is the site of various types of primary and metastatic tumors [14]. Ocular manifestations in canine multicentric lymphoma, which are the second most common clinical signs after lymph node enlargement, have been reported to occur in $37 \%$ of cases $[4,9,11]$. The main ocular manifestations related to lymphoma have been reported to be anterior uveitis (in 18\% of cases), retinal hemorrhage $(9 \%)$, panuveitis $(5 \%)$, posterior uveitis (3\%), and ocular adnexal involvement (2\%) [4]. Ocular adnexal involvement, which includes conjunctival infiltrates or keratitis consistent with corneal infiltrates of neoplastic lymphocytes, has rarely been reported in dogs.

This case series describes the ocular clinical signs, results of cytologic examinations and ocular ultrasonography, and treatments in dogs with multicentric lymphoma and corneoconjunctival involvement.

An 8-year-old neutered male Shih Tzu (C1) presented with a 10-day history of bilateral conjunctival hyperemia and chemosis. A 5-year-old neutered male Maltese (C2) presented with ocular discharge and conjunctival hyperemia. Neuro-ophthalmic examinations including menace response were normal for both. A 10-year-old neutered male Maltese (C3) presented with a 3-week history of blepharospasm, conjunctival hyperemia, and corneal edema. Menace response, dazzle, and pupillary light reflexes were absent in both eyes (oculi uterque [OU]). Tear production test (Schirmer tear test [STT]; Schering, USA) values were $15 \mathrm{~mm} / \mathrm{min}$ in the right eye (oculus dexter [OD]) and $17 \mathrm{~mm} / \mathrm{min}$ in the left eye (oculus sinister [OS]), and intraocular pressure (IOP) values obtained via rebound tonometer (TonoVet; iCare, Finland) were 14OD/11OS mmHg for C1. For C2 and C3, STT values were 18OD/17OS and 19OD/17OS $\mathrm{mm} / \mathrm{min}$ and IOP values were 12OD/13OS and 9OD/7OS mmHg, respectively. Slit-lamp biomicroscopy revealed severe conjunctival thickening, moderate hyperemia in both eyes (panels $\mathrm{A}^{-} \mathrm{C}$ in Fig. 1). Perilimbal or generalized corneal edema and neovascularization with negative fluorescein staining and aqueous flare to hypopyon were observed. The ocular fundus examination result was blurred in $\mathrm{C} 1$.

Tissue samples for cytologic examination were obtained from the conjunctiva by fine-needle aspiration (FNA) and scraping in $\mathrm{C} 1$. Cytologic analysis revealed $>90 \%$ mediumsized to large-sized lymphocytes and a few desquamated 


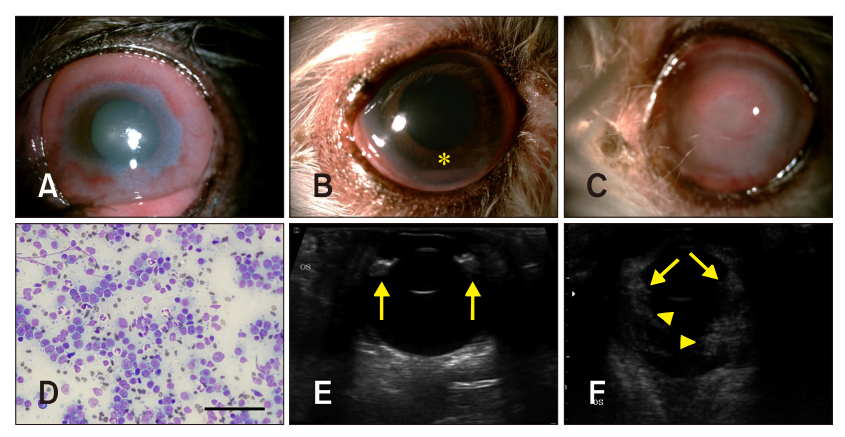

Fig. 1. Clinical appearance on initial presentation and the diagnostic approaches used. (A) The left eye in C1 (an 8-year-old neutered male Shih Tzu). (B) The right eye in C2 (a 5-year-old neutered male Maltese). (C) The left eye in C3 (a 10-year-old neutered male Maltese). Conjunctival thickening and hyperemia with corneal perilimbal (C1 and C2) or generalized (C3) edema and neovascularization were observed. Hypopyon (asterisk) was also seen in C2 (B). (D) The cytologic appearance of a fine-needle aspirate from conjunctiva in $\mathrm{C} 1$. Medium-sized to large-sized lymphocytes with malignant changes were detected. $400 \times$. Scale bar $=100 \mu \mathrm{m}$. (E and F) Respective B-scan ultrasound images for C1 and C3. Thickening of the ciliary body (arrows) and echogenic masses within the vitreous body (arrowheads) with vitreous degeneration were evident.

epithelial cells with moderate to high cellularity. The lymphocytes showed a moderately increased amount of cytoplasm and cytoplasmic basophilia. Many prominent nucleoli and granular or reticular nuclear chromatin were detected, along with mild to moderate anisokaryosis (panel D in Fig. 1). The ocular cytology was consistent with a diagnosis of lymphoma and the FNA sample results from enlarged superficial lymph nodes. All three dogs were diagnosed as having Stage $\mathrm{V}$ multicentric lymphoma, which was confirmed by cytological, radiographic, and ultrasonographic findings. Imaging studies showed enlarged abdominal lymph nodes, hepatomegaly, and splenomegaly. Ocular B-scan ultrasonography revealed ciliary body thickening with a ciliary body thickness of $2.74 \mathrm{~mm}$ in $\mathrm{C} 1$ (panel $\mathrm{E}$ in Fig. 1) and echogenic masses within vitreous in $\mathrm{C} 3$ (panel F in Fig. 1).

A subconjunctival injection of triamcinolone (Udenolon; Kukje, Korea) in combination with gentamicin (Gentapro; Huons, Korea) as an antibiotic prophylaxis was administered. Topical neomycin-polymyxin B-dexamethasone (Maxitrol; Alcon, USA), atropine (1\% Isopto-Atropine; Alcon), sodium hyaluronate (Lacure; Samil, Korea), and/or cyclosporine (Optimmune; Schering) were prescribed. C3 was also treated with oral L-VCAMP (L-asparaginase, vincristine, cyclophosphamide, doxorubicin, methotrexate, and prednisone) chemotherapy. In $\mathrm{C} 1$ and $\mathrm{C} 2$, the owners refused the offer of systemic chemotherapy.

After one month $(\mathrm{C} 1)$ and 3 weeks (C3), there were improvements to the conjunctival thickening and uveitis. The
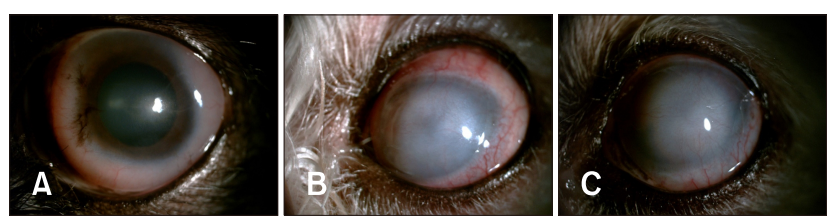

Fig. 2. Clinical appearance on follow-up examination. (A) The left eye in C1 (an 8-year-old neutered male Shih Tzu) after one month; the perilimbal corneal involvement improved and the conjunctival thickening and hyperemia also subsided. (B and C) The left eye in C3 (a 10-year-old neutered male Maltese) after 3 and 7 weeks, respectively; the conjunctival thickening subsided and the corneal edema and neovascularization also decreased. The corneoconjunctival lesions improved over time despite persistent corneal fibrosis.

perilimbal or generalized corneal involvement also improved and the corneas became more transparent (panels A and B in Fig. 2). Results of both eyes of ocular fundus examinations were normal in $\mathrm{C} 1$, with ocular fundus blurred before treatment because of the axial edema obscuring the visual axis. In $\mathrm{C} 3$, the menace response became positive in the right eye but was still negative in the left eye. The IOP values were $15 \mathrm{OD} / 4 \mathrm{OS} \mathrm{mmHg}$. The treatment was continued and 3 weeks later hyphema developed the left eye with an IOP of $10 \mathrm{OD} / 12 \mathrm{OS} \mathrm{mmHg}$. Four weeks later, the hyphema subsided with an IOP of 15OD/10OS $\mathrm{mmHg}$. The corneoconjunctival lesions improved further despite persistent corneal fibrosis (panel C in Fig. 2). Unfortunately, C2 was unable to be followed up.

Multicentric lymphoma accounts for approximately $80 \%$ of all lymphomas in dogs [13]. The majority of dogs are asymptomatic, but $20 \%$ may show nonspecific clinical signs, including anorexia, weight loss, vomiting, diarrhea, ascites, dyspnea, and fever [2]. Multicentric lymphoma classically presents with superficial lymphadenopathy, characterized by painless, rubbery, and discrete generalized enlarged lymph nodes [12,13]. The most common causes of superficial lymphadenopathy are infection and lymphoma. Physical examination, complete blood count, serum chemistry, urinalysis, and radiography are required in the initial evaluation of a patient with lymphadenopathy. Although anemia, thrombocytopenia, neutrophilia, hypercalcemia, and/or proteinuria might be observed in patients with multicentric lymphoma, cytologic examination using FNA or biopsy may be essential to confirm the diagnosis [13].

In our series, severe conjunctival thickening and hyperemia with corneal extension were detected on slit-lamp biomicroscopy. Because of their similar macroscopic appearance, conjunctival thickening must be distinguished from chemosis. For differentiation of the causes of conjunctival thickening, cytologic examination is recommended [14]. Of the numerous methods available, FNA is the most rapid, straightforward, safe, and minimally invasive method $[7,10]$. While various inflammatory 
cells tend to be seen in samples of conjunctivitis, multicentric lymphoma is composed of a monotonous population of medium-sized to large-sized lymphoid cells with occasional mitotic figures, as in this case series. A "starry-sky" appearance, whereby many apoptotic cells are present and macrophages contain apoptotic neoplastic lymphocytes known as tingible body macrophages, may also be observed [9]. Cytologic examination of FNA samples can distinguish between lymphoma and a benign lesion such as lymphocytic-plasmacytic conjunctivitis [1].

Ocular involvement, of the anterior uvea in particular, has been reported frequently in dogs with multicentric lymphoma $[3,14]$. Ocular changes in multicentric lymphoma commonly include infiltration of lymphoid cells and thickening of the uvea. Retinal detachment, posterior synechia, hypopyon, glaucoma, and hyphema may also be observed [11]. However, extraocular manifestations of lymphoma have rarely been reported in dogs, despite many cases in humans [7]. Further, reports of conjunctival involvement are very rare $[8,14]$. Corneal lymphoma has been reported to be associated with advanced (Stage V) systemic lymphoma and is located in the intrastromal or episcleral tissues, but it is not painful unless the lesion is ulcerated [6]. Corneoconjunctival thickening can induce a friable surface and incomplete eyelid closure (lagophthalmos), leading to a painful ulcer. In our cases, the corneoconjunctival lesions and cellular infiltrations subsided after local ophthalmic therapies.

Treatment of multicentric lymphoma generally requires systemic chemotherapy. Without treatment, the life expectancy of canine patients with Stage V lymphoma is 4 to 6 weeks $[5,13]$. Fortunately, most lymphomas are chemosensitive, and systemic chemotherapy is effective in $60 \%$ to $90 \%$ of dogs [12]. Combination chemotherapy protocols are known to be more effective than single-agent chemotherapy [12]; moreover, treatment can be tailored to the stage at presentation and the organs involved [14]. Local ophthalmic steroid therapy can be effective for alleviating ocular signs and can maintain a good quality of life by decreasing the corneal infiltration in the involved eye even without systemic chemotherapy, as in C1. In $\mathrm{C} 3$, topical therapy allowed a return of vision by decreasing the corneal infiltration in the right eye and might have helped prevent the hyphema from progressing to painful glaucoma in the left eye. In addition, local ophthalmic therapy might be the only treatment option in a lymphoma patient with exacerbated systemic conditions, where systemic treatment cannot be tolerated.

However, if evidence of malignancy is not found in the peripheral lymph nodes, the possibility of primary conjunctival lymphoma with no systemic involvement, which is classified as presumed solitary ocular lymphoma (PSOL), can be considered [5]. Surgical excision of PSOL using enucleation has been recommended to prevent metastasis and has the benefits of increasing survival time and relieving the pain caused by secondary ocular diseases, particularly glaucoma and/or uveitis $[5,8,14]$.

In conclusion, conjunctival involvement in multicentric lymphoma should be considered as the differential diagnosis when conjunctival thickening is detected on ophthalmic examination. If infiltration of neoplastic lymphoid cells is observed on cytologic evaluation of the conjunctiva, FNA of peripheral lymph nodes should be performed. Evidence of malignancy in peripheral lymph nodes suggests a diagnosis of systemic multicentric lymphoma [12].

\section{Acknowledgments}

This study was supported through BK21 PLUS Program for Creative Veterinary Science Research and the Research Institute for Veterinary Science (RIVS) of Seoul National University, Republic of Korea.

\section{Conflict of Interest}

The authors declare no conflicts of interest.

\section{References}

1. Comazzi S, Gelain ME. Use of flow cytometric immunophenotyping to refine the cytological diagnosis of canine lymphoma. Vet J 2011, 188, 149-155.

2. Ettinger SN. Principles of treatment for canine lymphoma. Clin Tech Small Anim Pract 2003, 18, 92-97.

3. Hendrix DVH. Diseases and surgery of the canine anterior uvea. In: Gelatt KN, Gilger BC, Kern TJ (eds.). Veterinary Ophthalmology. 5th ed. pp. 1146-1198, Wiley-Blackwell, Ames, 2013.

4. Krohne SG, Henderson NM, Richardson RC, Vestre WA. Prevalence of ocular involvement in dogs with multicentric lymphoma: prospective evaluation of 94 cases. Vet Comp Ophthalmol 1994, 4, 127-135.

5. Lanza MR, Musciano AR, Dubielzig RD, Durham AC. Clinical and pathological classification of canine intraocular lymphoma. Vet Ophthalmol 2018, 21, 167-173.

6. Ledbetter EC, Gilger BC. Diseases and surgery of the canine cornea and sclera. In: Gelatt KN, Gilger BC, Kern TJ (eds.). Veterinary Ophthalmology. 5th ed. pp. 976-1049, WileyBlackwell, Ames, 2013.

7. Maggs DJ. Conjunctiva. In: Maggs DJ, Miller PE, Ofri R (eds.). Slatter's Fundamentals of Veterinary Ophthalmology. 5th ed. pp. 140-154, Elsevier Saunders, St. Louis, 2013.

8. Olbertz L, Lima L, Langohr I, Wemer J, Teixeira L, Montiani-Ferreira F. Supposed primary conjunctival lymphoma in a dog. Vet Ophthalmol 2013, 16 (Suppl 1), 100-104.

9. Ota-Kuroki J, Ragsdale JM, Bawa B, Wakamatsu N, Kuroki K. Intraocular and periocular lymphoma in dogs and cats: a retrospective review of 21 cases (2001-2012). Vet Ophthalmol 
2014, 17, 389-396.

10. Stewart CJ, Duncan JA, Farquharson M, Richmond J. Fine needle aspiration cytology diagnosis of malignant lymphoma and reactive lymphoid hyperplasia. J Clin Pathol 1998, 51, 197-203.

11. Swanson JF. Ocular manifestations of systemic disease in the dog and cat: recent developments. Vet Clin North Am Small Anim Pract 1990, 20, 849-867.

12. Vail DM, Dobson JM. Tumours of the haemopoietic system and spleen. In: Dobson JM, Lascelles BDX (eds.). BSAVA
Manual of Canine and Feline Oncology. 3rd ed. pp. 285-308, British Small Animal Veterinary Association, Gloucester, 2010.

13. Vail DM, Pinkerton ME, Young KM. Hematopoietic tumors. In: Withrow SJ, Vail DM, Page RL (eds.). Withrow and MacEwen's Small Animal Clinical Oncology. 5th ed. pp. 608-678, Saunders, Philadelphia, 2013.

14. Vascellari M, Multari D, Mutinelli F. Unicentric extranodal lymphoma of the upper eyelid conjunctiva in a dog. Vet Ophthalmol 2005, 8, 67-70. 